\title{
Recognition of Off-Line Handwritten Devanagari Characters using Combinational Feature Extraction
}

\author{
Deepali R. Birajdar \\ Department of Electronics and Telecommunication \\ SVERI'S College of Engineering Pandharpur- \\ Pandharpur
}

\author{
Manasi M.Patil, Ph.D \\ Department of Electronics and Telecommunication \\ SVERI'S College of Engineering, \\ Pandharpur
}

\begin{abstract}
Handwritten Devanagari off-line characters recognition is a challenging task due to peculiarities involved in the writing styles of different person. We are collected features from 64 dimensional feature extraction techniques and shadow features for the Devanagari character recognition in this proposed scheme. These calculated features are used for further classification. Features which are used here for recognition means histograms of direction chain code of the contour points of the characters [4]. The features are classified using neural network and selected the best result by weighted majority voting technique.
\end{abstract}

\section{General Terms}

The general terms are Neural Network classifier is used with MLP classifier.

\section{Keywords}

64 dimensional features, Shadow features, neural network and weighted majority voting technique.

\section{INTRODUCTION}

The recognition of handwritten characters has been a popular research area from many years because of its various applications in various fields. In world Devanagari script is third most widely used script, used for several major languages such as Hindi, Sanskrit, Marathi and Nepali, and is used by more than 500 million people [1]. From different scripts unconstrained Devanagari writing is more complex than English cursive due to the possible variations in the order, number, direction and shape of the constituent strokes. Devanagari script has 50 characters which can be written as individual symbols in a word (some shown in figure 1). Because of presence of multiple loops, conjuncts, upper and lower modifiers and the number of disconnected and multi stroke characters the Devanagari Character recognition is becomes most complicated task [3],

\begin{tabular}{|l|l|l|l|l|l|l|}
\hline अ & आ & ई & ई & उ & अ & त्ई \\
\hline लृ & उ & इे & औ & औ & अं & अ० \\
\hline
\end{tabular}

\begin{tabular}{|c|c|c|c|c|c|c|}
\hline क & ख & ग & छ & So & व & $\varepsilon$ \\
\hline$G$ & झ- & भ & अ & ट & ठ & S \\
\hline ढ & UT & त & थ & $\mathrm{Cl}_{2}$ & धि & न \\
\hline प & 45 & ब & भ & म & य & 2 \\
\hline ल & व & 21 & $b p$ & स & ह & छब \\
\hline
\end{tabular}

Fig.1. Samples of handwritten Devanagari basic characters (a) Vowels, (b) Consonants
In 1977 the First research report was published on handwritten Devanagari Characters [6] but not more research work is done after that. Now a day's researchers have started to work on handwritten Devanagari characters and few research reports are also published. One of them is, Hanmandlu and Murthy [7, 10] proposed Fuzzy model based recognition of handwritten Hindi numerals and characters and they obtained $92.67 \%$ accuracy for Handwritten Devanagari numerals and $90.65 \%$ accuracy for Handwritten Devanagari characters. In this paper, we propose a scheme for unconstrained off-line handwritten Devanagari character recognition based on the feature obtained from shadow features and 64 dimensional features. Here in 64 dimensional feature extraction the bounding box of a character is segmented into 16 blocks and chain code histogram is computed in each block. These calculated features are then fed to the classifier for recognition. Here neural network is used for recognition. Rest of the paper is organized as follows. In Section 2 we discuss about Devanagari language data collection and the preprocessing of the data used for this scheme. In Section 3 Feature extraction procedures are presented. In Section 4 discussions about recognition classifier. In section 5 Results obtained after application of the technique on handwritten Devanagari characters are shown. Conclusion on the paper is given in Section 6 .

\section{PROPOSED SCHEME}

In our proposed scheme first we are collected the handwritten data from different writer's . These data samples are same as shown in figure 1.This collected data is further proposed for normalization.

\subsection{Data Collection}

All the collected handwritten data from different writers are in a paper document form. After that it is scanned using optical scanning and stored in a file of picture elements, called pixels. Because of the writing styles of different person, characters can have different shapes. So that recognition of unconstrained handwritten characters becomes a difficult task. So we have to resize the image convert it in to bitmap file by preprocessing as given below. Here in for recognition we have collected 1080 samples.

\subsection{Prepossessing}

Here for scanning a flat bed scanner is used. This image is jpg color image it has been converted into grayscale image and then to two-tone ( 0 and 1$)$ images (Here ' 1 ' represents object point and ' 0 ' represents background point) it is threshold binary image. For removing noises from the images, we have used a method discussed in [5].We has to calculate all top, bottoms, left and right boundaries to resize the no of pixels which are exact multiple of 4 for simplification purpose. 


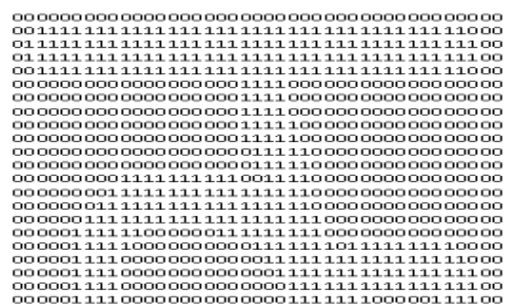

Fig 2.Binary image

\section{FEATURE EXTRACTION}

In the following we are given a brief description of the two feature sets used in our proposed multiple classifier system. How the shadow features are extracted from scaled bitmapped character image and 64 dimensional features for our recognition purpose. The feature extraction techniques are described below.

\subsection{Shadow Features of character}

For computing shadow features [8], the rectangular boundary enclosing the character image is divided into eight octants, for each octant shadow of character segment is computed on two perpendicular sides so a total of 16 shadow features are obtained. Shadow is basically the length of the projection on the sides as shown in figure 3.These features are computed on scaled image. For this we have to make eight windows from paint.
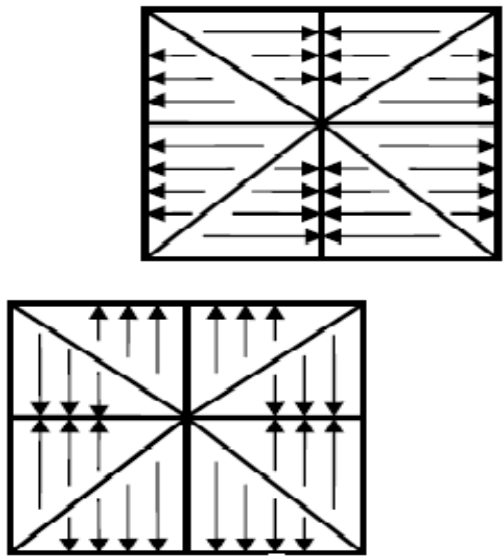

Fig.3. Shadow Features

\subsection{Dimensional Feature Extraction}

The scaled image should have pixel size in exact multiple of 4 , which is used for finding out the features. First we have to find out the contour points of the image by using the Canny method. The Canny method finds edges by looking for local maxima of the gradient of I. The gradient is calculated using the derivative of a Gaussian filter. The method uses two thresholds, to detect strong and weak edges, and includes the weak edges in the output only if they are connected to strong edges. This method detected true weak edges as shown in fig.3.
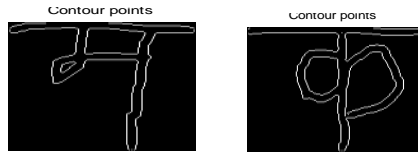

Fig.4 It shows contour points

This above bonding box is divided into $4 \times 4$ blocks which results into 16 blocks. Then for each block 4 dimensional features are computed. In each of these blocks, the direction chain code for each contour point is noted and the frequency of the direction codes is computed. Here we use chain code of four directions only [directions 0 (horizontal), 1 (45 degree slanted), 2(vertical) and 3 (135 degree slanted)]. See Fig 4(b) for illustration of four chain code directions.

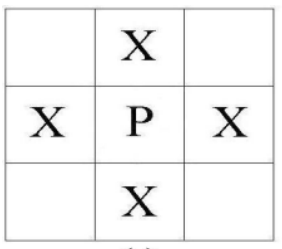

(a)

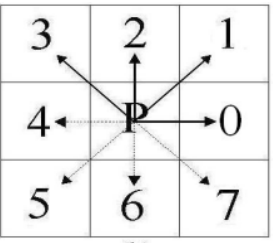

(b)
Fig. 5. (a) For a point $P$ and its four neighbors are shown by ' $X$ ',(b) For a point $P$ the direction codes for its eight neighboring points are shown.

We assume chain code of direction 0 and 4,1 and 5, 2 and 6 , 3 and 7, are same. Thus in each block we get an array of four integer values representing the frequencies and those frequency values are used as feature. Histogram of the values of these four direction codes in each block of a Devanagari character is shown in Fig. 5 (d).Thus, for $4 \times 4$ blocks $4 \times 4 \times 4=64$ features are computed. To normalize the features we compute maximum value of the histograms from all the blocks. We divide each of the above features by this maximum value to get the feature values between 0 and 1.For example one sample of chain code and its count shown in fig.5(e)

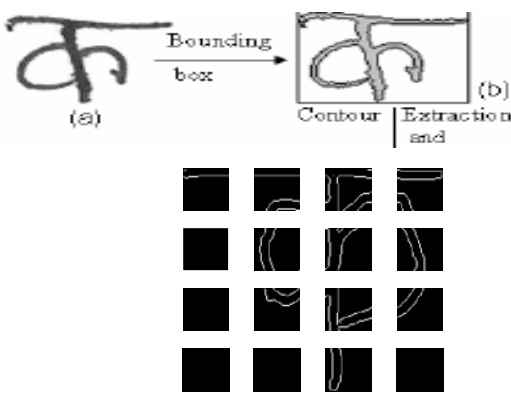

(c)

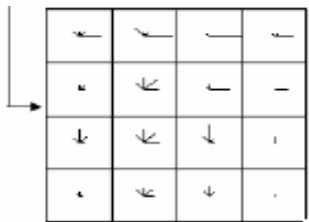

(d)64 diamensional features

\begin{tabular}{|c|c|}
\hline Chaix.Coda & Cont \\
\hline 0 & 1 \\
\hline 1 & 0 \\
\hline 2 & $\mathbf{1 1}$ \\
\hline 3 & 3 \\
\hline
\end{tabular}

(e)

Fig.6. Pictorial representation of the 64 dimensional feature extraction process for a sample Devanagari character. (a) Two tone image of a Devanagari character, (b) Bounding box of the character. (c) Contour of the character shown in black color and the bounding box is segmented into $4 \mathrm{X} 4$ blocks. (d) Chain code histogram (e) sample chain code and its count. 
The chain code for the character contour will yield a smooth, unbroken curve as it grows along the perimeter of the character and completely encompasses the character. When a character has multiple connections, then there can be multiple chain codes to represent the contour of the character. From this algorithm we are computing features from single character, if we have to compute features from compound character then we have first segmented that particular character by using another algorithm .That process is called as segmentation [9].

\section{DEVNAGARI CHARACTER RECOGNITION}

In our proposed scheme we used the same MLP with 3 layers including one hidden layer for above two feature sets consisting of 64 dimensional features and 16 shadow features we get the experimental results using these features for recognition of handwritten Devanagari characters is presented as below. In this paper we are used the non-compound and single characters so no segmentation is required.

This classifier is trained with standard back propagation algorithm [11]. Which is minimizes the errors that are sum of squared errors for the training samples by conducting a gradient descent search in the weight space. Numbers of neurons in input layer of MLPs are 64 and 16 for 64 dimensional features and shadow features respectively. Number of neurons in Hidden layer is not fixed, we experimented on the values in between 20-70 for getting optimal result and finally it is set 32 and 30 for 64 dimensional and shadow features respectively. The output layer contained one node for each class., so the number of neurons in output layer is 49. And classification was accomplished by a simple maximum response strategy.

\subsection{Classifier Combination}

For better classification performance we have to design pattern recognition system. This objective can be achieved by development of different classification scheme for any pattern recognition problem to be solved. It had been observed in such design studies, that although one of the designs would yield the best performance, the sets of patterns misclassified by the different classifiers would not necessarily overlap. There are different classifiers designs are available for potentially offered complementary information about the pattern to be classified which could be harnessed to improve the performance of the selected classifier. So instead of depending on a single decision making scheme we can combine classifiers for best performance. Combination of individual classifier outputs overcomes deficiencies of features and of single classifiers. We can combine outputs from several classifiers to produce a more accurate result. Combination of classifier takes two forms: combination of like classifiers trained on different data sets and combination of dissimilar classifiers. We have two similar neural networks classifiers as discussed above, which are trained on 16 shadow features, 64 dimensional features respectively. The resultant outputs are assurance associated with each class. As these outputs cannot be compared directly, we used an aggregation function for combining the results of all two classifiers. Our strategy is based on weighted majority voting scheme as described below.

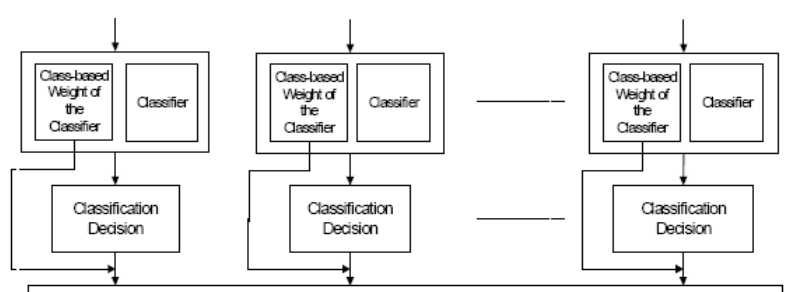

Decision Fusion: Counting Class-besed Weighted Votes for Individual Cass Decisions to Support a Final Decision

Final Decision

Fig.7 weighted majority voting scheme for combining classifires.

So if kth classifier decision to assign the unknown pattern to the ith class is denoted by Uik with $1 \leq \mathrm{i} \leq \mathrm{c}$, c being the number of classes, then the final combined decision di com supporting assignment to the ith class takes the form of :-

$$
\begin{aligned}
& d \mathbf{i}^{\text {com }}=\Sigma \text { rk * Uik .......1 } \leq \mathbf{i} \leq c \\
& k=1,2
\end{aligned}
$$

The final decision $d \mathbf{c o m}$ is therefore :-

$$
\begin{aligned}
& d^{\text {com }}=\max d \mathbf{i}^{\text {com }} \\
& \mathbf{1} \leq \mathbf{i} \leq c \\
& \text { rk }=\frac{\mathbf{f}_{\mathbf{k}}}{\sum_{\mathrm{k}=1}^{2} \mathrm{f}_{\mathrm{k}}}
\end{aligned}
$$

Where $\mathrm{c}=35$ and $\mathrm{r} 1, \mathrm{r} 2$ are respectively 0.626 and 0.373 as f1> f2

f1 $=74.40 \%$ result of classifier trained with 64 dimensional features.

f2 $=44.30 \%$ result of classifier trained with Shadow features.

\section{RESULTS}

In our proposed scheme we are collected the handwritten Devanagari character dataset from different handwrites. For preparation of the training and test sets a sample set of 1080 handwritten Devanagari characters are considered. A training set of 756 samples and test set of 162 samples are formed. Results are shown in table 2 . We considered top 1 choice, top 2 choices, top 3 choices, top 4 choices and top 5 choices for classification results. We applied 3 -fold cross validation testing. We divided the whole dataset into three parts. In first fold, first two parts are used for training and third part is used for testing. In a second fold, second and third part is used for training and first part is used for testing. In fold three, first and third part is used for training and second part is used for testing We used the same MLP for three different feature sets as discussed above in section 3 . The results of three MLPs are shown below. 
Table 1. Results of three different MLP

\begin{tabular}{|c|c|c|c|c|}
\hline MLP & $\begin{array}{l}\text { Input } \\
\text { layer } \\
\text { Neuro } \\
\text { n }\end{array}$ & $\begin{array}{l}\text { Hidden } \\
\text { Layer } \\
\text { Neuron }\end{array}$ & $\begin{array}{l}\text { Output } \\
\text { Layer } \\
\text { Neuron }\end{array}$ & Result \\
\hline $\begin{array}{l}64 \\
\text { dimensional } \\
\text { feature based }\end{array}$ & 64 & 32 & 49 & $74.40 \%$ \\
\hline $\begin{array}{l}\text { Shadow } \\
\text { Feature based }\end{array}$ & 16 & 30 & 49 & $44.30 \%$ \\
\hline
\end{tabular}

Table 2. Top Choices Results

\begin{tabular}{|l|l|l|}
\hline \multicolumn{1}{|c|}{ Sr. No. } & \multicolumn{1}{|c|}{ Proposed method result } & $\begin{array}{c}\text { Accuracy } \\
\text { obtained }\end{array}$ \\
\hline 1 & Top 1 choices & $79.10 \%$ \\
\hline 2 & Top 2 choices & $81.33 \%$ \\
\hline 3 & Top 3 choices & $83.32 \%$ \\
\hline 4 & Top 4 choices & $87.55 \%$ \\
\hline 5 & Top 5 choices & $99.00 \%$ \\
\hline
\end{tabular}

From survey we know that the technique which giving $80.71 \%$ accuracy, which is better than results reported by Kumar, Singh[4] and N. Sharma, U. Pal, F.Kimura, S. Pal [5]. We used 4900 samples dataset which is larger than 4750 used by M. Hanmandlu, O.V. R. Murthy, V.K. Madasu[10]. They reported $69.78 \%$ overall recognition rate for handwritten Devanagari characters which is similar to our top 1 choices result. Their coarse classification is giving $90.65 \%$ accuracy but we have obtained $99.99 \%$ accuracy.

Table 3. Recognition success

\begin{tabular}{|c|c|c|}
\hline Sr. No. & Method proposed by & $\begin{array}{c}\text { Accuracy } \\
\text { obtained }\end{array}$ \\
\hline 1 & Kumar and Singh [4] & $80 \%$ \\
\hline 2 & $\begin{array}{c}\text { N. Sharma, U. Pal, F. } \\
\text { Kimura, and S. Pal [5] }\end{array}$ & $80.36 \%$ \\
\hline 3 & $\begin{array}{c}\text { M. Hanmandlu, O.V. R. } \\
\text { Murthy, V.K.Madasu[7] }\end{array}$ & $90.65 \%$ \\
\hline 4 & Proposed method & $99.00 \%$ \\
\hline
\end{tabular}

\section{CONCLUSION}

From this paper we conclude that an off-line handwritten Devnagari character recognition system consisting of two different features using simple feed forward Multilayer Perceptrons. We obtained encouraging results. This technique can be applied on handwritten Devnagari numerals also and it will also be helpful for research towards other similar Indian scripts like Tamil, Bengali, Gujarati, Gurmukhi, Oriya.

\section{ACKNOWLEDGMENTS}

Our thanks to the experts who have contributed towards development of the template.

\section{REFERENCES}

[1] Sandhya Arora, Debotosh Bhattacharjee, Mita Nasipuri, "Combining Multiple Feature Extraction Techniques for Handwritten Devnagari Character Recognition", IEEE Region 10 Colloquium and the Third ICIIS, Kharagpur,2008.

[2] Nei Kato,Shin'ichiro Omachi,Hirotomo Aso, Yoshiaki Nemoto "A Handwritten Character Recognition System Using Directional Element Feature and Asymmetric Mahalanobis Distance", IEEE transactions on pattern analysis and machine intelligence, vol. 21 , no. 3, march 1999 pp 258-262

[3] L. Koerich ,Large Vocabulary off-line handwritten word recognition. PhD thesis, Ecole de Technologic Superieure, Montreal-Canada, August 2002.

[4] Satish Kumar and Chandan Singh, "A Study of Zernike Moments and its use in Devnagari Handwritten Character Recognition", Intl.Conf. on Cognition and Recognition, pp. 514-520, 2005.

[5] N. Sharma, U. Pal*, F. Kimura**, and S. Pal," Recognition of Off-Line Handwritten Devnagari Characters Using Quadratic Classifier", Computer Vision and Pattern Recognition, pp. 805 - 816, 2006.

[6] I. K. Sethi, and B. Chatterjee, Machine recognition of constrained hand printed devnagari, Pattern Recognition, vol. 9, pp. 69-75, 1977.

[7] M. Hanmandlu and O.V. Ramana Murthy, "Fuzzy Model Based Recognition of Handwritten Hindi Numerals", Intl.Conf. on Cognition and Recognition, pp. 490-496, 2005.

[8] Sandhya Arora1. Debotosh Bhattacharjee2, Mita Nasipuri2, L. Malik4 "Performance Comparison of SVM and ANN for Handwritten Devnagari Character Recognition", International Journal of Computer Science Issues, Vol. 7, Issue 3, No 6, May 2010.

[9] Vikas J Dongre Vijay H Mankar," A Review of Research on Devnagari Character Recognition", International Journal of Computer Applications (0975 - 8887)Volume 12- No.2, November 2010.

[10] M. Hanmandlu, O.V. Ramana Murthy, Vamsi Krishna Madasu, "FuzzyModel based recognition of Handwritten Hindi characters", IEEE Computer society, Digital Image Computing Techniques and Applications , 2007.

[11] J. Hertz, A. Krogh, R.G. Palmer, "An Introduction to neural Computation", Addison-Wesley (1991). 